\title{
Tectonic Evolution of the Bering Shelf-Chukchi Sea-Arctic Margin and Adjacent Landmasses, GSA Special Paper 360
}

\author{
Lithostratigraphic, conodont, and other fossil links between lower Paleozoic strata in northern \\ and central Alaska and northeastern Russia, \\ by Julie A. Dumoulin, Anita G. Harris, Mussa Gagiev, Dwight C. Bradley, and John E. Repetski, \\ Chapter 16.
}

\section{Appendix 1. Uppermost Cambrian and Ordovician conodont faunas from northeast Russia (mainly from the Kolyma-Omolon superterrane).}

All conodont collections except no. 88 were made by Mussa Gagiev; sample no. 88 was collected by Jaime Toro, (Department of Geology and Geography, West Virginia University, Morgantown, WV).

The collections were analyzed by Anita G. Harris and John E. Repetski and are housed in the conodont laboratory of the U.S. Geological Survey National Center, Reston, Virginia, U.S.A.

Mussa Gagiev died before providing precise locality data for most of these collections. The locality data given below were taken from a variety of sources including notes accompanying or written on the microfossil slides (mostly in Russian). Thus, the stratigraphic, geographic, and structural settings given below for most of these collections are generalized or incomplete. Nonetheless, these data can be used for small-scale Ordovician paleogeographic reconstructions as all samples are known to come from the Kolyma-Omolon superterrane. We have tried, without success, to obtain copies of Gagiev's field notes; we hope that the field data will eventually be provided by his colleagues at the Russian Academy of Sciences, Siberian Branch, Magadan, Russia.

Abbreviations of conodont faunal affinities: CO, cosmopolitan realm; TC, tropical cosmopolitan realm; S, Siberian province; SA, Siberian-Alaskan (species restricted to Siberia, peri-Siberia, and Alaska); LS, Laurentian-Siberian (species occur in Laurentia, periLaurentia, Siberia, and peri-Siberia); NCS, North China-Siberia (species restricted to North China, Siberia, and peri-Siberia); NCSA, North China-Siberia-Alaska (species known only from North China, Siberia, peri-Siberia, and Alaska); INDET., (specimens too poorly preserved for generic assignment and thus, faunal affinity).

Faunal realm, province, or mixed affinities listed in order of decreasing representation.

Conodont abundance (includes all identified elements of a multielement apparatus); R, rare ( $<5$ specimens); C, common (5-<20 specimens); A, abundant (>20 specimens); indets., indeterminate bar, blade, platform, and coniform element fragments (specimens too poorly preserved for generic assignment). 


\begin{tabular}{|c|c|c|c|c|c|}
\hline $\begin{array}{c}\text { SAMPLE NO. } \\
\text { (CONODONT } \\
\text { FAUNAL } \\
\text { AFFINITIES) } \\
\end{array}$ & $\begin{array}{l}\text { SECTION AND } \\
\text { FIELD NO. }\end{array}$ & $\begin{array}{c}\text { STRATIGRAPHIC } \\
\text { UNIT, LITHOLOGY, } \\
\text { AND STRUCTURAL } \\
\text { BLOCK } \\
\end{array}$ & CONODONT FAUNA & AGE & CAI \\
\hline $\begin{array}{l}1 \\
\text { (CO, NCS, } \\
\text { S, SA, TC) }\end{array}$ & $\begin{array}{l}* 417-18 \text { VPK } \\
(1983 r) O_{2} \\
\text { Koperanko? }\end{array}$ & & $\begin{array}{l}\text { Acanthodina sp. indet. [R] } \\
\text { Complexodus? sp. indet. (all fragments) [C] } \\
\text { Drepanodus sp. indet. [C] } \\
\text { Histiodella holodentata Ethington \& Clark [R] } \\
\text { Parapanderodus? consimilis (Moskalenko) [R] } \\
\text { Paroistodus sp. indet. [R] } \\
\text { Phragmodus cf. P. flexuosus Moskalenko [C] } \\
\text { Polonodus sp. indet. [R] } \\
\text { Protopanderodus cf. P. varicostatus (Sweet \& } \\
\text { Bergström) [C] } \\
\text { Tangshanodus tangshanensis An [C] } \\
\text { unassigned coniform elements [C] } \\
\text { indets. [C] }\end{array}$ & $\begin{array}{l}\text { early Llanvirn } \\
\text { (Eo. suecicus } \\
\text { Zone) }\end{array}$ & 4 \\
\hline $\begin{array}{l}2 \\
(\mathrm{CO})\end{array}$ & $\begin{array}{l}\text { Shul'gipa } \\
\text { Nezabudka } \\
\text { Cr. *4-12 } \\
(1982 \text { r) O2 }\end{array}$ & $\begin{array}{l}\text { Omulevka uplift } \\
\text { Omolon terrane }\end{array}$ & $\begin{array}{l}\text { Periodon sp. indet. of post-Pe. flabellum } \\
\text { morphotype [R] } \\
\text { Protopanderodus sp. indet. [R] }\end{array}$ & $\begin{array}{l}\text { latest Arenig- } \\
\text { Caradoc }\end{array}$ & $\begin{array}{c}\sim 3.5 \\
\text { (adventitiou } \\
\text { s organic } \\
\text { matter }\end{array}$ \\
\hline $\begin{array}{l}3 \\
\text { (INDET.) }\end{array}$ & $\begin{array}{l}\text { Shul'gipa } \\
\text { Nezabudka } \\
\text { Cr. *1278-4 } \\
\text { (1980-82rr) }\end{array}$ & Omolon terrane & indets. [C] & $\begin{array}{l}\text { Probably Early } \\
\text { Ordovician }\end{array}$ & 3 or 4 \\
\hline $\begin{array}{l}4 \\
(\mathrm{CO})\end{array}$ & \begin{tabular}{|l|} 
Shul'gipa \\
$* 1278-5$ \\
$(1980-82 \mathrm{rr})$ \\
\end{tabular} & Omolon terrane & Protopanderodus sp. indet. [R] & $\begin{array}{l}\text { middle Arenig or } \\
\text { younger } \\
\text { Ordovician }\end{array}$ & $\sim 4$ \\
\hline $\begin{array}{l}5 \\
(\mathrm{CO})\end{array}$ & $\begin{array}{l}\text { Shul'gipa } \\
\text { Nezabudka } \\
\text { Cr. *2/6 } \\
(1982), O_{2-3}\end{array}$ & Omolon terrane & $\begin{array}{l}\text { Periodon sp. indet. }[\mathrm{R}] \\
\text { indets. }[\mathrm{R}]\end{array}$ & $\begin{array}{l}\text { latest Arenig- } \\
\text { middle Caradoc }\end{array}$ & 3.5 \\
\hline $\begin{array}{l}6 \\
(\mathrm{CO})\end{array}$ & $\begin{array}{l}\text { Shul'gipa } \\
\text { Nezabudka } \\
\text { Cr. *4/5 } \\
(1982)\end{array}$ & Omolon terrane & $\begin{array}{l}\text { Periodon sp. indet. [R] } \\
\text { Prioniodus sp. indet. [R] } \\
\text { indets. [R] }\end{array}$ & $\begin{array}{l}\text { latest Arenig- } \\
\text { Llanvirn }\end{array}$ & 4 \\
\hline
\end{tabular}




\begin{tabular}{|c|c|c|c|c|c|}
\hline $\begin{array}{l}7 \\
(\mathrm{CO}, \mathrm{TC})\end{array}$ & $\begin{array}{l}\text { Shul'gipa } \\
* 1673-8 \\
(1984)\end{array}$ & Omolon terrane & $\begin{array}{l}\text { Oepikodus sp. indet. [R] } \\
\text { Parapanderodus striatus (Graves \& Ellison) [R] } \\
\text { Paroistodus cf. P. parallelus (Lindström) [R] } \\
\text { Protoprioniodus cf. P. aranda Cooper [R] } \\
\text { Tripodus sp. [R] } \\
\text { unassigned drepanodontid [R] } \\
\text { indets. [R] }\end{array}$ & $\begin{array}{l}\text { late Arenig-earliest } \\
\text { Llanvirn }\end{array}$ & 4.5 \\
\hline $\begin{array}{l}8 \\
(\mathrm{LS}, \mathrm{CO})\end{array}$ & $\begin{array}{l}* 2428-3 \\
(1980-82 \mathrm{rr}) \\
\mathrm{O}_{2-3}-\mathrm{S}_{1}\end{array}$ & & $\begin{array}{l}\text { Drepanoistodus sp. [R] } \\
\text { Oistodus multicorrugatus Harris [R] } \\
\text { indets. [R] }\end{array}$ & $\begin{array}{l}\text { latest Arenig-early } \\
\text { Llanvirn (T. laevis } \\
\text { Zone-Hi. sinuosa } \\
\text { Zone) }\end{array}$ & $\sim 4$ \\
\hline $\begin{array}{l}9 \\
(\mathrm{CO})\end{array}$ & $\begin{array}{l}\text { Shul'gipa } \\
* 2209-11 \\
(1980-82 \mathrm{rr}) \\
\text { O3 }\end{array}$ & Omolon terrane & $\begin{array}{l}\text { Periodon sp. indet. of post-Pe. flabellum } \\
\text { morphotype [R] } \\
\text { indets. [R] }\end{array}$ & $\begin{array}{l}\text { latest Arenig- } \\
\text { Caradoc }\end{array}$ & $\sim 3$ \\
\hline $\begin{array}{l}10 \\
(\mathrm{CO})\end{array}$ & $\begin{array}{l}* 2428-4 \\
(1980-82 \mathrm{rr}) \\
\mathrm{S}_{1}\end{array}$ & & $\begin{array}{l}\text { Drepanoistodus } \mathrm{sp} .[\mathrm{R}] \\
\text { Jumudontus ganada Cooper [R] } \\
\text { Parapanderodus striatus (Graves \& Ellison) [R] } \\
\text { indets. [C] }\end{array}$ & $\begin{array}{l}\text { late Arenig }(R . \\
\text { andinus Zone- } T \text {. } \\
\text { laevis Zone) }\end{array}$ & $\sim 4$ \\
\hline $\begin{array}{l}11 \\
\text { (SA) }\end{array}$ & $\begin{array}{l}\text { Shul'gipa } \\
* 2503 \\
(1980-83 \mathrm{rr}) \\
\text { O3 }\end{array}$ & Omolon terrane & $\begin{array}{l}\text { Acanthocordylodus sp. indet. [R] } \\
\text { Stereoconus corrugatus Moskalenko [R] } \\
\text { indets. [C] }\end{array}$ & $\begin{array}{l}\text { Llanvirn-early } \\
\text { Caradoc(?) }\end{array}$ & $\sim 4$ \\
\hline $\begin{array}{l}12 \\
(\mathrm{CO})\end{array}$ & $\begin{array}{l}\text { Shul'gipa } \\
(1983 \mathrm{r}) \\
\mathrm{O}_{2}\end{array}$ & Omolon terrane & $\begin{array}{l}\text { Parapanderodus striatus (Graves \& Ellison) [R] } \\
\text { Periodon sp. indet. [R] } \\
\text { indets. [C] }\end{array}$ & $\begin{array}{l}\text { late Arenig-early } \\
\text { Llanvirn }\end{array}$ & 4 \\
\hline $\begin{array}{l}13 \\
(\mathrm{CO}, \mathrm{SA} \\
\mathrm{TC}, \mathrm{LS})\end{array}$ & $\begin{array}{l}* 830-5 a \\
(1983 r) \\
\mathrm{O}_{2}\end{array}$ & & $\begin{array}{l}\text { Acanthocordylodus sp. [C] } \\
\text { Drepanoistodus sp. [R] } \\
\text { Histiodella holodentata Ethington \& Clark [R] } \\
\text { Juanognathus variabilis Serpagli [R] } \\
\text { Oistodus multicorrugatus Harris [R] } \\
\text { Protopanderodus cf. P. rectus (Lindström) [C] } \\
\text { Parapanderodus striatus (Graves \& Ellison) [C] } \\
\text { indets. [A] }\end{array}$ & $\begin{array}{l}\text { early Llanvirn (Hi. } \\
\text { holodentata Zone) }\end{array}$ & $\sim 4$ \\
\hline
\end{tabular}




\begin{tabular}{|c|c|c|c|c|c|}
\hline $\begin{array}{l}14 \\
(\mathrm{CO}, \mathrm{LS})\end{array}$ & $\begin{array}{l}* 830-5 b \\
(1983 r) \\
\text { Oar2-3 }\end{array}$ & & $\begin{array}{l}\text { Protopanderodus spp. [A] } \\
\text { Oistodus multicorrugatus Harris [C] } \\
\text { Periodon sp. indet. [R] } \\
\text { Spinodus spinatus (Hadding) [R] }\end{array}$ & $\begin{array}{l}\text { latest Arenig-early } \\
\text { Llanvirn (T. laevis } \\
\text { Zone-Hi. sinuosa } \\
\text { Zone) }\end{array}$ & $\sim 4$ \\
\hline $\begin{array}{l}15 \\
(\mathrm{CO}, \mathrm{LS})\end{array}$ & $\begin{array}{l}418-6 \\
(1984 r)\end{array}$ & & $\begin{array}{l}\text { Drepanoistodus sp. [C] } \\
\text { Protopanderodus } \mathrm{n} . \mathrm{sp} .1 \text { or } 2 \text { of Repetski, } \\
1982 \text { [R] } \\
\text { Scolopodus floweri Repetski [C] } \\
\text { Scolopodus kelpi Repetski [C] }\end{array}$ & $\begin{array}{l}\text { M. dianae Zone } \\
\text { (latest Tremadoc- } \\
\text { earliest Arenig) }\end{array}$ & $\begin{array}{l}5 \text { and minor } \\
5.5\end{array}$ \\
\hline $\begin{array}{l}16 \\
(\mathrm{CO})\end{array}$ & $\begin{array}{l}443-1 \\
(1984 r)\end{array}$ & & Protopanderodus sp. [R] & Early Ordovician & 5 \\
\hline $\begin{array}{l}17 \\
\text { (INDET.) }\end{array}$ & $\begin{array}{l}443-7 \\
(1984 r) \\
\end{array}$ & Sjapjakine River & unassigned Ordovician coniform elements [R] & Ordovician & 4.5 \\
\hline $\begin{array}{l}18 \\
\text { (INDET.) }\end{array}$ & $\begin{array}{l}* 443-22, \mathrm{O}_{2} \\
(1984 \mathrm{r})\end{array}$ & Sjapjakine River & drepanodontiform $[\mathrm{R}]$ & Ordovician & 4.5 \\
\hline $\begin{array}{l}19 \\
(\mathrm{CO}, \mathrm{LS})\end{array}$ & $\begin{array}{l}444-28 \\
(1984)\end{array}$ & & $\begin{array}{l}\text { Drepanoistodus sp. [R] } \\
\text { Protopanderodus robustus (Hadding) [C] } \\
\text { Oistodus multicorrugatus Harris [R] } \\
\text { multicostate scolopodid or stereoconid }[\mathrm{R}] \\
\text { indets. [C] }\end{array}$ & $\begin{array}{l}\text { latest Arenig-early } \\
\text { Llanvirn (T. laevis } \\
\text { Zone-Hi. sinuosa } \\
\text { Zone) }\end{array}$ & $4.5-5$ \\
\hline $\begin{array}{l}20 \\
(\mathrm{CO})\end{array}$ & $\begin{array}{l}444-29 \\
(1984)\end{array}$ & & $\begin{array}{l}\text { Drepanoistodus sp. [R] } \\
\text { Periodon aculeatus Hadding [A] } \\
\text { Protopanderodus rectus (Lindström) [R] } \\
\text { unassigned element fragments [C] } \\
\text { indets. [C] }\end{array}$ & $\begin{array}{l}\text { latest Arenig- } \\
\text { earliest Caradoc }\end{array}$ & 5 \\
\hline $\begin{array}{l}21 \\
(\mathrm{CO}, \mathrm{SA})\end{array}$ & $\begin{array}{l}444-29 \\
\text { Cunskuup } \\
\text { (1984) }\end{array}$ & & $\begin{array}{l}\text { Periodon flabellum (Lindström) trans. to Pe. } \\
\text { aculeatus Hadding [C] } \\
\text { Protopanderodus } \mathrm{sp} \text {. [R] } \\
\text { unassigned Sc? }(3 \text { morphotypes) elements of } \\
\text { pre-Acanthocordylodus morphotype [R] } \\
\text { indets. [R] }\end{array}$ & late Arenig & 5 \\
\hline
\end{tabular}




\begin{tabular}{|c|c|c|c|c|c|}
\hline $\begin{array}{l}22 \\
(\mathrm{CO}, \mathrm{S}, \\
\mathrm{NCS})\end{array}$ & $\begin{array}{l}445-3 \\
(1984 r)\end{array}$ & & $\begin{array}{l}\text { Drepanoistodus sp. indet. [R] } \\
\text { Paroistodus sp. indet. [R] } \\
\text { Periodon flabellum (Lindström) trans. to Pe. } \\
\text { aculeatus Hadding [R] } \\
\text { Tangshanodus tangshanensis An [R] } \\
\text { Plectodina? sp. of Siberian affinities [R] } \\
\text { Protopanderodus cf. P. graeai Hamar [C] } \\
\text { unassigned M elements [R] } \\
\text { indets. [C] }\end{array}$ & $\begin{array}{l}\text { early Llanvirn }(\mathrm{Hi} \text {. } \\
\text { sinuosa Zone- } \mathrm{Hi} \text {. } \\
\text { holodentata Zone) }\end{array}$ & 5 \\
\hline $\begin{array}{l}23 \\
(\mathrm{CO}, \mathrm{S}, \mathrm{SA}, \\
\mathrm{LS})\end{array}$ & $\begin{array}{l}\text { Shul'gipa } \\
\text { Syayakinye } \\
445-9 \\
(1984)\end{array}$ & Omolon terrane & $\begin{array}{l}\text { Acanthocordylodus? sp. [R] } \\
\text { Oistodus multicorrugatus Harris [R] } \\
\text { Periodon sp. indet. of post-Pe. flabellum } \\
\text { morphotype [R] } \\
\text { Protopanderodus sp. indet. [R] } \\
\text { unassigned erismodids? of Siberian aspect [C] } \\
\text { unassigned coniform (3 morphotypes) [R] } \\
\text { indets. [A] }\end{array}$ & $\begin{array}{l}\text { latest Arenig }(T \text {. } \\
\text { laevis Zone- } H i \text {. } \\
\text { sinuosa Zone })\end{array}$ & $\sim 4$ \\
\hline $\begin{array}{l}24 \\
(\mathrm{CO}, \mathrm{LS}, \mathrm{S})\end{array}$ & $\begin{array}{l}445-11 \\
\text { Cenekune } \\
(1984)\end{array}$ & & $\begin{array}{l}\text { Cornuodus? sp. [R] } \\
\text { Erraticodon } \mathrm{sp} \text {. indet. [R] } \\
\text { Histiodella } \mathrm{n} \text {. sp. } 2 \text { of Harris and others (1979) } \\
{[\mathrm{R}]} \\
\mathrm{M} \text { and } \mathrm{Sc} \text { fragments of a Siberian plectodinid? } \\
{[\mathrm{R}]} \\
\text { Protopanderodus sp. indet. [R] } \\
\text { unassigned M elements (3 morphotypes) [R] } \\
\text { unassigned coniform fragments [R] }\end{array}$ & $\begin{array}{l}\text { middle Llanvirn } \\
\text { (Ph. polonicus } \\
\text { Zone ) }\end{array}$ & 4 \\
\hline $\begin{array}{l}25 \\
(\mathrm{CO}, \mathrm{NCS})\end{array}$ & $\begin{array}{l}445-29 \\
(1984 r)\end{array}$ & & $\begin{array}{l}\text { Tangshanodus tangshanensis An? [R] } \\
\text { Protopanderodus } \mathrm{sp} .[\mathrm{R}] \\
\text { indets. [C] }\end{array}$ & $\begin{array}{l}\text { early Llanvirn }(\mathrm{Hi} \text {. } \\
\text { sinuosa Zone- } \mathrm{Hi} \text {. } \\
\text { holodentata Zone) }\end{array}$ & 4 \\
\hline $\begin{array}{l}26 \\
(\mathrm{CO}, \mathrm{S})\end{array}$ & $\begin{array}{l}446-26 \\
1984\end{array}$ & & $\begin{array}{l}\text { Drepanodus sp. [C] } \\
\text { robust hyaline digyrate Siberian apparatus (cf. } \\
\text { Trichonodella spp. of Moskalenko, 1973) [C] } \\
\text { Juanognathus? sp. indet. [R] } \\
\text { Periodon aculeatus Hadding? [R] } \\
\text { Periodon? sp. indet. [R] } \\
\text { Protopanderodus sp. indet. [R] } \\
\text { indets. [A] }\end{array}$ & Llanvirn & 4 \\
\hline
\end{tabular}




\begin{tabular}{|c|c|c|c|c|c|}
\hline \begin{tabular}{|l|}
27 \\
$(\mathrm{CO})$
\end{tabular} & $\begin{array}{l}\text { Shul'gipa } \\
\text { Krelekh- } \\
\text { Sienye } \\
460-2 \\
1984 \mathrm{r}\end{array}$ & Omolon terrane & $\begin{array}{l}\text { Periodon aculeatus Hadding (late form) }[\mathrm{R}] \\
\text { Protopanderodus } \mathrm{sp} .[\mathrm{R}] \\
\text { indets. }[\mathrm{R}]\end{array}$ & $\begin{array}{l}\text { Llanvirn-early } \\
\text { Caradoc }\end{array}$ & 3.5 \\
\hline \begin{tabular}{|l|}
28 \\
$(\mathrm{CO})$
\end{tabular} & $\begin{array}{l}460-4 \\
1984\end{array}$ & & $\begin{array}{l}\text { Drepanoistodus sp. indet. [R] } \\
\text { Periodon aculeatus Hadding }[\mathrm{R}] \\
\text { indets. [R] }\end{array}$ & $\begin{array}{l}\text { latest Arenig-early } \\
\text { Caradoc }\end{array}$ & 3 \\
\hline \begin{tabular}{|l|}
29 \\
$(\mathrm{CO})$
\end{tabular} & $\begin{array}{l}464-9 \\
1984\end{array}$ & & $\begin{array}{l}\text { Periodon aculeatus Hadding }[\mathrm{R}] \\
\text { Polonodus tablepointensis Stouge }[\mathrm{C}] \\
\text { Protopanderodus varicostatus (Sweet \& } \\
\text { Bergström) }[\mathrm{C}] \\
\text { unassigned M elements }[\mathrm{R}] \\
\text { Spinodus spinatus (Hadding) }[\mathrm{R}] \\
\text { indets. }[\mathrm{C}]\end{array}$ & early Llanvirn & 4 \\
\hline $\begin{array}{l}30 \\
\text { (TC) }\end{array}$ & $\begin{array}{l}351-2 \\
\text { Korago } \\
(1976 \mathrm{r}) \mathrm{O}_{2}\end{array}$ & $\begin{array}{l}\text { Omolon massif, } \\
\text { Narzan Formation. } \\
\text { Saturn and } \\
\text { Astronomicheskaya } \\
\text { Rivers interfluve. } \\
\text { Omolon terrane } \\
\end{array}$ & $\begin{array}{l}\text { Rossodus manitouensis Repetski \& Ethington } \\
{[\mathrm{C}]} \\
\text { indets. }[\mathrm{A}]\end{array}$ & $\begin{array}{l}\text { middle Tremadoc } \\
\text { (Rossodus } \\
\text { manitouensis } \\
\text { Zone) }\end{array}$ & $2.5-3$ \\
\hline $\begin{array}{l}31 \\
\text { (TC) }\end{array}$ & $\begin{array}{l}\text { Tkonenko } \\
150-10 \\
(1983 r)\end{array}$ & & $\begin{array}{l}\text { Acanthodus sp. indet. [R] } \\
\text { Rossodus manitouensis Repetski \& Ethington } \\
{[\mathrm{C}]} \\
\text { Variabiloconus bassleri (Furnish) [C] } \\
\text { indets. [C] }\end{array}$ & $\begin{array}{l}\text { middle Tremadoc } \\
\text { (Rossodus } \\
\text { manitouensis } \\
\text { Zone) }\end{array}$ & 3 \\
\hline \begin{tabular}{|l|}
32 \\
$(\mathrm{CO}, \mathrm{TC})$
\end{tabular} & $\begin{array}{l}279 / 10 \\
(1983 \mathrm{r})\end{array}$ & & $\begin{array}{l}\text { Cordylodus intermedius Furnish [R] } \\
\text { unassigned coniform elements [R] } \\
\text { Teridontus nakamurai (Nogami) [R] } \\
\text { indets. [R] }\end{array}$ & $\begin{array}{l}\text { latest Late } \\
\text { Cambrian (lower } \\
\text { Cordylodus } \\
\text { proavus Zone-C. } \\
\text { intermedius Zone) }\end{array}$ & $\sim 5.5-6$ \\
\hline \begin{tabular}{|l}
33 \\
(INDET.)
\end{tabular} & $\begin{array}{l}167 \\
(1967)\end{array}$ & & \begin{tabular}{|l|} 
drepanodontiform $[\mathrm{R}]$ \\
unassigned coniform $[\mathrm{R}]$
\end{tabular} & $\begin{array}{l}\text { Early-Middle } \\
\text { Ordovician, } \\
\text { probably Early }\end{array}$ & 2 or 3 \\
\hline $\begin{array}{l}34 \\
(\mathrm{CO}, \mathrm{LS})\end{array}$ & 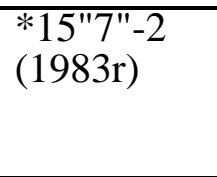 & & $\begin{array}{l}\text { Coleodus } \mathrm{sp} .[\mathrm{R}] \\
\text { Oistodus multicorrugatus Harris }[\mathrm{R}] \\
\text { Protopanderodus sp. [R] } \\
\text { indets. [R] }\end{array}$ & $\begin{array}{l}\text { late Arenig-earliest } \\
\text { Llanvirn (T. laevis } \\
\text { Zone-Hi. sinuosa } \\
\text { Zone) }\end{array}$ & 4 \\
\hline
\end{tabular}




\begin{tabular}{|c|c|c|c|c|c|}
\hline $\begin{array}{l}35 \\
(\mathrm{TC}, \mathrm{CO})\end{array}$ & $\begin{array}{l}* 355-1 \\
(1983) \\
\text { O1 }\end{array}$ & & $\begin{array}{l}\text { Cordylodus } \mathrm{sp} .[\mathrm{R}] \\
\text { Rossodus manitouensis } \text { Ethington \& Repetski } \\
{[\mathrm{C}]} \\
\text { Variabiloconus bassleri (Furnish) }[\mathrm{C}]\end{array}$ & $\begin{array}{l}\text { middle Tremadoc } \\
\text { (Rossodus } \\
\text { manitouensis } \\
\text { Zone) }\end{array}$ & 4 \\
\hline $\begin{array}{l}36 \\
(\mathrm{CO}, \mathrm{TC})\end{array}$ & $\begin{array}{l}* 355-2 \\
(1983 \mathrm{r}) \\
\mathrm{O}_{1} \mathrm{ar}\end{array}$ & & $\begin{array}{l}\text { Cordylodus sp. }[\mathrm{C}] \\
\text { Drepanodus pervetus Nowlan [C] } \\
\text { Variabiloconus bassleri (Furnish) [R] }\end{array}$ & $\begin{array}{l}\text { middle Tremadoc } \\
\text { (R. manitouensis } \\
\text { Zone?) }\end{array}$ & 4 \\
\hline $\begin{array}{l}37 \\
(\mathrm{CO})\end{array}$ & $\begin{array}{l}\text { Tkachenio(?) } \\
* 1529-4 \\
(1989) \\
\mathrm{O}_{2}\end{array}$ & & $\begin{array}{l}\text { Dapsilodus mutatus (Rhodes)? [R] } \\
\text { unassigned drepanodontiform [R] } \\
\text { unassigned Sc element }[\mathrm{R}]\end{array}$ & Caradoc-Ashgill & 4 \\
\hline $\begin{array}{l}38 \\
\text { (INDET.) }\end{array}$ & $\begin{array}{l}\text { Wasaut } \\
\text { 6727-1, O2Hr } \\
(1990)\end{array}$ & & unassigned robust hyaline drepanodontid? [R] & Ordovician & $4 ?$ \\
\hline $\begin{array}{l}39 \\
(\mathrm{NCS}, \mathrm{CO})\end{array}$ & $\begin{array}{l}\text { Wasonol } \\
9075-2 \\
\text { Iggor O } \\
\text { O2el } \\
\text { P. xapkuu } \\
\end{array}$ & & $\begin{array}{l}\text { Drepanodus sp. [C] } \\
\text { Tasmanognathus sishuiensis Zhang [C] } \\
\text { indets. [C] }\end{array}$ & $\begin{array}{l}\text { early Llanvirn }(\mathrm{Hi} \text {. } \\
\text { sinuosa Zone- } \mathrm{Hi} \text {. } \\
\text { holodentata Zone) }\end{array}$ & 4 \\
\hline $\begin{array}{l}40 \\
\text { (TC) }\end{array}$ & $\begin{array}{l}85-690 \\
\text { O1 } \\
\text { Kotokh } \\
\text { Yagodnovi }\end{array}$ & $\begin{array}{l}\text { Sonny Suite; Fore- } \\
\text { Kolyma uplift; } \\
\text { Kotokh-Balyk- } \\
\text { Takh Rivers, upper } \\
\text { course. } \\
\text { Prikolyma terrane }\end{array}$ & $\begin{array}{l}\text { Variabiloconus bassleri (Furnish) }[\mathrm{R}] \\
\text { deformed and shattered indet. coniform } \\
\text { fragments }[\mathrm{C}]\end{array}$ & Early Ordovician & $4.5 ?$ \\
\hline $\begin{array}{l}41 \\
(\mathrm{CO})\end{array}$ & $\begin{array}{l}85-690 \\
\mathrm{O}_{2}\end{array}$ & $\begin{array}{l}\text { Sonny Suite; Fore- } \\
\text { Kolyma uplift; } \\
\text { Kotokh-Balyk- } \\
\text { Takh Rivers, upper } \\
\text { course. } \\
\text { Prikolyma terrane }\end{array}$ & $\begin{array}{l}\text { Drepanoistodus sp. indet. [R] } \\
\text { Oulodus sp. indet. [R] } \\
\text { Periodon aculeatus Hadding [C] } \\
\text { Protopanderodus liripipus Kennedy, Barnes \& } \\
\text { Uyeno [C] } \\
\text { Walliserodus sp. [C] } \\
\text { indets. [A] }\end{array}$ & Caradoc-Ashgill & 4.5 \\
\hline $\begin{array}{l}42 \\
\text { (SA) }\end{array}$ & $\begin{array}{l}\text { 1390, O3ash } \\
\text { Shabanov } \\
(1990 r)\end{array}$ & $\begin{array}{l}\text { Dolghin Suite } \\
\text { Drevnja River, } \\
\text { Supkanja River } \\
\text { basin }\end{array}$ & Acanthodina sp. [R] & Llanvirn-Ashgill & 4 \\
\hline
\end{tabular}




\begin{tabular}{|c|c|c|c|c|c|}
\hline $\begin{array}{l}43 \\
(\mathrm{SA})\end{array}$ & $\begin{array}{l}\text { 1391, O3ash } \\
\text { Shabanov } \\
(1990 r)\end{array}$ & $\begin{array}{l}\text { Dolghin Suite } \\
\text { Drevnja R., } \\
\text { Supkanja River } \\
\text { basin }\end{array}$ & $\begin{array}{l}\text { Acanthodina sp. }[\mathrm{C}] \\
\text { robust coniform indets. }[\mathrm{R}]\end{array}$ & Llanvirn-Ashgill & 4 \\
\hline $\begin{array}{l}44 \\
\text { (SA, } \\
\text { C) }\end{array}$ & $\begin{array}{l}1394, \mathrm{O}_{3} \\
\text { Shabanov } \\
\text { (1990r) }\end{array}$ & $\begin{array}{l}\text { Irjudi Suite } \\
\text { Drevnjaja River } \\
\text { Omulevka uplift } \\
\text { Omulevka terrane }\end{array}$ & $\begin{array}{l}\text { Acanthodina sp. [C] } \\
\text { Acanthocordylodus sp. [R] } \\
\text { Panderodus sp. [R] } \\
\text { indets. [C] }\end{array}$ & Llanvirn-Ashgill & 4 \\
\hline $\begin{array}{l}45 \\
(\mathrm{CO})\end{array}$ & $\begin{array}{l}1678, \mathrm{O}_{2} \\
\text { Wasonol } \\
(1990 \mathrm{r})\end{array}$ & $\begin{array}{l}\text { Sonny Suite, } \\
\text { Khakodate R., } \\
\text { Omulevka uplift } \\
\text { Omulevka terrane }\end{array}$ & Drepanoistodus sp. indet. [R] & Llanvirn-Ashgill & 4 \\
\hline $\begin{array}{l}46 \\
\text { (NCS, } \\
\text { NCSA, LS) }\end{array}$ & $\begin{array}{l}2110-4 \\
\text { Shabanov } \\
\text { (1990r) }\end{array}$ & $\begin{array}{l}\text { Kharkindgha Suite, } \\
\text { Kharkindgha River, } \\
\text { Omulevka uplift } \\
\text { Omulevka terrane }\end{array}$ & $\begin{array}{l}\text { Histiodella cf. Hi. n. sp. } 2 \text { of Harris and others, } \\
1979 \text { [R] } \\
\text { Tangshanodus tangshanensis An [C] } \\
\text { Tasmanognathus sishuiensis Zhang [C] } \\
\text { indets. [C] }\end{array}$ & $\begin{array}{l}\text { early-middle } \\
\text { Llanvirn }\end{array}$ & 4 \\
\hline $\begin{array}{l}47 \\
(\mathrm{CO})\end{array}$ & $\begin{array}{l}690 \\
\text { phi cTO } \\
\text { Lichagin(?) }\end{array}$ & $\begin{array}{l}\text { Sonny Suite; Fore- } \\
\text { Kolyma uplift; } \\
\text { Kotokh-Balyk- } \\
\text { Takh Rivers, upper } \\
\text { course. } \\
\text { Prikolyma terrane } \\
\end{array}$ & Periodon aculeatus Hadding [R] & $\begin{array}{l}\text { late Arenig-early } \\
\text { Caradoc }\end{array}$ & $4-4.5$ \\
\hline $\begin{array}{l}48 \\
\text { (INDET.) }\end{array}$ & $\begin{array}{l}\mathrm{c} 666-2 \\
(1984 \mathrm{r}) \mathrm{O}_{2} \\
\text { Cepeyen } \\
\end{array}$ & & $\begin{array}{l}\text { unassigned M of Ordovician morphotype [R] } \\
\text { indets. [R] }\end{array}$ & Ordovician & 5 \\
\hline $\begin{array}{l}49 \\
\text { (INDET.) }\end{array}$ & $\begin{array}{l}\text { Mirney Creek } \\
1045-01\end{array}$ & Omulevka terrane & Siberian ansellid? [R] & $\begin{array}{l}\text { Middle or Late } \\
\text { Ordovician }\end{array}$ & 3 or less \\
\hline $\begin{array}{l}50 \\
(\mathrm{CO})\end{array}$ & $\begin{array}{l}1045-1 \\
\mathrm{O}_{2} \mathrm{~K}\end{array}$ & $\begin{array}{l}\text { Omulevka terrane if } \\
\text { this is also Mirney } \\
\text { Creek }\end{array}$ & $\begin{array}{l}\text { Periodon aculeatus Hadding [C] } \\
\text { Protopanderodus } \mathrm{sp.}[\mathrm{R}] \\
\text { unassigned coniform ( } 3 \text { morphotypes) }[\mathrm{R}] \\
\text { indets. [C] }\end{array}$ & $\begin{array}{l}\text { late Arenig-early } \\
\text { Caradoc }\end{array}$ & 5 \\
\hline
\end{tabular}




\begin{tabular}{|c|c|c|c|c|c|}
\hline $\begin{array}{l}51 \\
(\mathrm{CO})\end{array}$ & $1045-2$ & $\begin{array}{l}\text { Omulevka terrane if } \\
\text { this is also Mirney } \\
\text { Creek }\end{array}$ & $\begin{array}{l}\text { Panderodus sp. [R] } \\
\text { Paroistodus sp. indet. [R] } \\
\text { Periodon a culeatus Hadding or } \text { P. grandis } \\
\text { (Ethington) }[\mathrm{R}] \\
\text { unassigned coniforms }[\mathrm{R}] \\
\text { indet. }[\mathrm{C}]\end{array}$ & $\begin{array}{l}\text { latest Arenig- } \\
\text { Caradoc }\end{array}$ & 4.5 \\
\hline \begin{tabular}{|l|}
52 \\
$(\mathrm{CO})$
\end{tabular} & $\begin{array}{l}1045-4 \\
\mathrm{O}_{2} \mathrm{~K}\end{array}$ & $\begin{array}{l}\text { Omulevka terrane if } \\
\text { this is also Mirney } \\
\text { Creek }\end{array}$ & $\begin{array}{l}\text { Periodon aculeatus Hadding (M elements with at } \\
\text { least } 8 \text { anterior denticles indicating a post-Eo. } \\
\text { suecicus Zone Middle Ordovician age) }[\mathrm{R}] \\
\text { Drepanoistodus sp. indet. }[\mathrm{R}] \\
\text { indets. }[\mathrm{R}]\end{array}$ & \begin{tabular}{|l} 
Llanvirn-early \\
Caradoc
\end{tabular} & 4.5 \\
\hline \begin{tabular}{|l|}
53 \\
$(\mathrm{CO})$
\end{tabular} & $\begin{array}{l}1045-5 \\
\mathrm{O}_{2} \mathrm{~K}_{2}\end{array}$ & $\begin{array}{l}\text { Omulevka terrane if } \\
\text { this is also Mirney } \\
\text { Creek }\end{array}$ & $\begin{array}{l}\text { Periodon aculeatus Hadding (M elements with at } \\
\text { least } 6 \text { anterior denticles) [A] } \\
\text { Protopanderodus sp. [R] } \\
\text { indets. [R] }\end{array}$ & $\begin{array}{l}\text { Llanvirn-early } \\
\text { Caradoc }\end{array}$ & 4.5 \\
\hline \begin{tabular}{|l|}
54 \\
$(\mathrm{CO})$
\end{tabular} & $\begin{array}{l}1045-7 \\
\mathrm{O}_{2} \mathrm{~K}_{2}\end{array}$ & $\begin{array}{l}\text { Omulevka terrane if } \\
\text { this is also Mirney } \\
\text { Creek }\end{array}$ & Protopanderodus sp. indet. $[\mathrm{R}]$ & \begin{tabular}{|l|} 
Llanvirn-middle \\
Caradoc
\end{tabular} & 4.5 or 5 \\
\hline $\begin{array}{l}55 \\
(\mathrm{CO})\end{array}$ & $\begin{array}{l}\text { Kapara Creek } \\
1045-8 \\
\mathrm{O}_{2} \mathrm{~K}_{2}\end{array}$ & & $\begin{array}{l}\text { Periodon aculeatus Hadding }[\mathrm{R}] \\
\text { Protopanderodus sp. of late Arenig or younger } \\
\text { Middle Ordovician morphotype }[\mathrm{R}] \\
\text { unassigned coniform }[\mathrm{R}] \\
\text { indets. }[\mathrm{C}]\end{array}$ & $\begin{array}{l}\text { late Arenig-early } \\
\text { Caradoc }\end{array}$ & $\sim 4$ \\
\hline \begin{tabular}{|l|}
56 \\
$(\mathrm{CO})$
\end{tabular} & $\begin{array}{l}1045-9 \\
\mathrm{O}_{2} \mathrm{~K}_{2}\end{array}$ & & $\begin{array}{l}\text { drepanodontids }[\mathrm{R}] \\
\text { Panderodus sp. }[\mathrm{R}] \\
\text { Periodon } \text { sp. indet. }[\mathrm{R}]\end{array}$ & $\begin{array}{l}\text { Llanvirn-early } \\
\text { Caradoc }\end{array}$ & 4.5 \\
\hline \begin{tabular}{|l}
57 \\
$(\mathrm{SA}, \mathrm{CO})$
\end{tabular} & $\begin{array}{l}\text { 1045-23 } \\
\text { O3Ash }\end{array}$ & & $\begin{array}{l}\text { Acanthodina } \mathrm{sp} .[\mathrm{R}] \\
\text { Panderodus } \mathrm{sp} \text {. }[\mathrm{R}] \\
\text { Pseudooneotodus mitratus (Moskalenko) }[\mathrm{R}]\end{array}$ & $\begin{array}{l}\text { late Llanvirn- } \\
\text { Ashgill }\end{array}$ & $\sim 4.5$ \\
\hline $\begin{array}{l}58 \\
\text { (TC) }\end{array}$ & $\begin{array}{l}\text { 1045-25 } \\
\text { O3Ash }\end{array}$ & & $\begin{array}{l}\text { belodinids [R] } \\
\text { indets. }[\mathrm{R}]\end{array}$ & $\begin{array}{l}\text { late Llanvirn- } \\
\text { Ashgill }\end{array}$ & 5 \\
\hline \begin{tabular}{|l}
59 \\
(TC, LS)
\end{tabular} & $\begin{array}{l}\text { 1045-32 } \\
\text { O3Ash }\end{array}$ & & $\begin{array}{l}\text { belodinids }[\mathrm{C}] \\
\text { Belodina } \mathrm{cf} \text {. B. vulgaris Sweet }[\mathrm{R}] \\
\text { digyrate } \mathrm{P} \text { element }[\mathrm{R}] \\
\text { indets. }[\mathrm{R}]\end{array}$ & Ashgill & 5 \\
\hline
\end{tabular}




\begin{tabular}{|c|c|c|c|c|}
\hline $\begin{array}{l}60 \\
(\mathrm{SA})\end{array}$ & $\begin{array}{l}\text { 1045-41 } \\
\text { O3Ash }\end{array}$ & $\begin{array}{l}\text { Belodina? repens Moskalenko }[\mathrm{R}] \\
\text { unassigned hyaline ramiform element of } \\
\text { Siberian(?) morphotype }[\mathrm{R}]\end{array}$ & Ashgill & 3 \\
\hline $\begin{array}{l}61 \\
(\mathrm{TC})\end{array}$ & $\begin{array}{l}1045-53 \\
\text { Tonuya M } \\
\text { O3Ash }\end{array}$ & Belodina? sp. [R] & Ashgill(?) & 3 \\
\hline $\begin{array}{l}62 \\
(\mathrm{CO})\end{array}$ & $\begin{array}{l}89-449 \\
\text { O3tr }\end{array}$ & $\begin{array}{l}\text { Amorphognathus sp. indet. [R] } \\
\text { drepanodontiform }[\mathrm{R}] \\
\text { indets. }[\mathrm{R}]\end{array}$ & Caradoc-Ashgill & $4 ?$ \\
\hline $\begin{array}{l}63 \\
(\mathrm{CO})\end{array}$ & $\begin{array}{l}89-449 \\
\text { O3tr }\end{array}$ & Panderodus gracilis (Branson \& Mehl) [R] & $\begin{array}{l}\text { late Llanvirn- } \\
\text { Ashgill }\end{array}$ & $4 ?$ \\
\hline $\begin{array}{l}64 \\
(\mathrm{CO})\end{array}$ & $\begin{array}{l}89-455 \\
\text { O3tr }\end{array}$ & Pa fragment Amorphognathus? sp. indet. [R] & Caradoc-Ashgill & 3 or 4 \\
\hline $\begin{array}{l}65 \\
(\mathrm{CO})\end{array}$ & $89-461$ & Panderodus sp. [R] & Caradoc-Ashgill & 3 or 4 \\
\hline $\begin{array}{l}66 \\
(\mathrm{CO})\end{array}$ & $89-463$ & $\begin{array}{l}\text { Dapsilodus sp. }[\mathrm{C}] \\
\text { unassigned digyrate elements }[\mathrm{C}] \\
\text { indets. [C] }\end{array}$ & Caradoc-Silurian & 3 or 4 \\
\hline $\begin{array}{l}67 \\
(\mathrm{CO})\end{array}$ & $\begin{array}{l}89-475 \\
\text { S }_{1 \mathrm{mt}}\end{array}$ & $\begin{array}{l}\text { Drepanodus sp. indet. (redeposited?) [R] } \\
\text { Oulodus sp. indet. [R] } \\
\text { Panderodus sp. [C] } \\
\text { unassigned coniform [R] } \\
\text { indets. [C] }\end{array}$ & $\begin{array}{l}\text { Possibly Silurian } \\
\text { with redeposited } \\
\text { Ordovician } \\
\text { conodonts }\end{array}$ & 3 or 4 \\
\hline $\begin{array}{l}68 \\
(\mathrm{CO})\end{array}$ & $\begin{array}{l}89-519 \\
\text { INa } \\
\text { O3tr }\end{array}$ & $\begin{array}{l}\text { Drepanoistodus sp. indet. [R] } \\
\text { Panderodus gracilis (Branson \& Mehl) [R] } \\
\text { Protopanderodus insculptus (Branson \& Mehl) } \\
{[\mathrm{R}]}\end{array}$ & middle-late Ashgill & 3 or 4 \\
\hline $\begin{array}{l}69 \\
(\mathrm{CO})\end{array}$ & $\begin{array}{l}\text { V.F. Belyui } \\
47-23 \\
\text { (1978r phi) }\end{array}$ & $\begin{array}{l}\text { Periodon aculeatus Hadding [C] } \\
\text { Protopanderodus } \mathrm{sp} . \quad[\mathrm{C}] \\
\text { indets. [R] }\end{array}$ & $\begin{array}{l}\text { late Arenig-early } \\
\text { Caradoc, likely } \\
\text { late Arenig-early } \\
\text { Llanvirn }\end{array}$ & 3 or 4 \\
\hline $\begin{array}{l}70 \\
(\mathrm{CO})\end{array}$ & $\begin{array}{l}\text { V.F. Belyui } \\
47-23 \\
(1978 r) \\
\text { R. Kharitoni } \\
\mathrm{O}_{2-3}\end{array}$ & $\begin{array}{l}\text { Paroistodus sp. indet. [R] } \\
\text { Periodon aculeatus Hadding [A] } \\
\text { Protopanderodus sp. indet. [C] } \\
\text { unassigned coniform elements [R] } \\
\text { indets. [A] }\end{array}$ & $\begin{array}{l}\text { late Arenig-early } \\
\text { Caradoc }\end{array}$ & 4 \\
\hline
\end{tabular}




\begin{tabular}{|c|c|c|c|c|c|}
\hline $\begin{array}{l}71 \\
(\mathrm{NCS}, \mathrm{SL} \\
\mathrm{CO})\end{array}$ & $\begin{array}{l}5694-4 \mathrm{~A} \\
\text { pie-90 } \\
\text { O2lln1 } \\
(1980)\end{array}$ & & $\begin{array}{l}\text { Hi. holodentata Ethington \& Clark? [R] } \\
\text { Tangshanodus tangshanensis An (chiefly } \\
\text { fragments) [C] } \\
\text { stereoconids (deformed) }[\mathrm{R}] \\
\text { indets. }[\mathrm{A}]\end{array}$ & $\begin{array}{l}\text { early Llanvirn } \\
\text { (lower Hi. } \\
\text { holodentata } \\
\text { Zone?) }\end{array}$ & $5-6$ \\
\hline $\begin{array}{l}72 \\
\text { (NCS) }\end{array}$ & \begin{tabular}{|l|}
6309 \\
-90 \\
$\mathrm{O}_{2}$ Llandeilo? \\
$(1980 \mathrm{r})$
\end{tabular} & & $\begin{array}{l}\text { Tangshanodus tangshanensis An [C] } \\
\text { Siberian-North Chinese erismodids }[\mathrm{C}] \\
\text { indets. [A] }\end{array}$ & \begin{tabular}{|l} 
early Llanvirn \\
\end{tabular} & 8 \\
\hline $\begin{array}{l}73 \\
(\mathrm{TC}, \mathrm{LS})\end{array}$ & \begin{tabular}{|l|} 
Kopara \\
$751-1$
\end{tabular} & & $\begin{array}{l}\text { Colaptoconus quadraplicatus (Branson \& Mehl } \\
\text { [C] } \\
\text { Clavohamulus n. sp. cf. C. n. sp. A of } \\
\text { Repetski, } 1982 \text { [C] }\end{array}$ & $\begin{array}{l}\text { latest Tremadoc- } \\
\text { earliest Arenig } \\
\text { (Low diversity } \\
\text { Zone-M. dianae } \\
\text { Zone) }\end{array}$ & Mixed, 1-6 \\
\hline $\begin{array}{l}74 \\
(\mathrm{LS})\end{array}$ & $\begin{array}{l}751-1 \\
(1976 r) \\
\mathrm{O}_{1}\end{array}$ & & $\begin{array}{l}\text { Clavohamulus n. sp. cf. C. n. sp. A of } \\
\text { Repetski, } 1982 \text { [R] } \\
\text { Juanognathus? felicitii (Ji \& Barnes) [R] }\end{array}$ & $\begin{array}{l}\text { M. dianae Zone } \\
\text { (latest Tremadoc- } \\
\text { earliest Arenig) }\end{array}$ & Mixed, 1-6 \\
\hline \begin{tabular}{|l|}
75 \\
$(\mathrm{TC}, \mathrm{CO})$
\end{tabular} & \begin{tabular}{|l}
$796 / 4$ \\
$\mathrm{O}_{1}$
\end{tabular} & & $\begin{array}{l}\text { "Acanthodus" lineatus (Furnish) [C] } \\
\text { Cordylodus angulatus Pander [R] } \\
\text { Rossodus manitouensis Repetski \& Ethington } \\
{[\mathrm{C}]} \\
\text { Variabiloconus bassleri (Furnish) }[\mathrm{A}]\end{array}$ & $\begin{array}{l}\text { middle Tremadoc } \\
\text { (R. manitouensis } \\
\text { Zone) }\end{array}$ & 3 or 4 \\
\hline $\begin{array}{l}76 \\
(\mathrm{CO})\end{array}$ & \begin{tabular}{|l|}
$605, \mathrm{O}_{1}$ \\
Shamin \\
Birkachanshii
\end{tabular} & $\begin{array}{l}\text { Narzan Formation, } \\
\text { right side of } \\
\text { Nakuchan R. (left } \\
\text { of Omolon R.) } \\
\text { Omolon massif } \\
\text { Omolon terrane }\end{array}$ & $\begin{array}{l}\text { Teridontus nakamurai (Nogami) }[\mathrm{R}] \\
\text { indets. }[\mathrm{R}]\end{array}$ & $\begin{array}{l}\text { latest Cambrian- } \\
\text { early Early } \\
\text { Ordovician }\end{array}$ & 4 \\
\hline $\begin{array}{l}77 \\
(\mathrm{CO})\end{array}$ & $\begin{array}{l}\text { A-43-5A, } \\
\text { Likman } \\
\mathrm{O}_{2}\end{array}$ & & $\begin{array}{l}\text { Panderodus } \mathrm{sp} \text {. indet. (deformed) [R] } \\
\text { Periodon } \mathrm{sp} \text {. indet. [R] } \\
\text { Prioniodus } \mathrm{sp} \text {. indet. }[\mathrm{R}] \\
\text { Protopanderodus } \mathrm{sp} \text {. indet. [R] }\end{array}$ & $\begin{array}{l}\text { Llanvirn-middle } \\
\text { Caradoc }\end{array}$ & $5-5.5$ \\
\hline $\begin{array}{l}78 \\
(\mathrm{CO})\end{array}$ & $\begin{array}{l}\mathrm{A}-609-3 \mathrm{~A}, \\
\mathrm{O}_{2}\end{array}$ & & Dapsilodus sp. indet. [R] & \begin{tabular}{|l|} 
Llanvirn-Ashgill \\
\end{tabular} & 4.5 \\
\hline
\end{tabular}




\begin{tabular}{|c|c|c|c|c|c|}
\hline \begin{tabular}{|l}
79 \\
$(\mathrm{CO})$
\end{tabular} & $\begin{array}{l}\text { C*40a } \\
(1983 r) \\
\text { Sturidoila- } \\
\text { *epe }\end{array}$ & & $\begin{array}{l}\text { Drepanodus sp. }[\mathrm{C}] \\
\text { Oepikodus evae }(\text { Lindström) [A] } \\
\text { Protopanderodus } \mathrm{sp} .[\mathrm{R}]\end{array}$ & $\begin{array}{l}\text { middle Arenig ( } O . \\
\text { evae Zone) }\end{array}$ & 2 \\
\hline $\begin{array}{l}80 \\
(\mathrm{TC}, \mathrm{CO})\end{array}$ & $\begin{array}{l}\text { C*40b } \\
(1983 r) \\
\text { Sturidoila- } \\
\text { *epe }\end{array}$ & & $\begin{array}{l}\text { Oepikodus communis (Ethington \& Clark) [A] } \\
\text { Oepikodus evae (Lindström) [C] } \\
\text { Paroistodus } \mathrm{sp} .[\mathrm{R}] \\
\text { Protopanderodus } \mathrm{sp} .[\mathrm{R}]\end{array}$ & $\begin{array}{l}\text { middle Arenig ( } O . \\
\text { evae Zone) }\end{array}$ & 2 \\
\hline $\begin{array}{l}81 \\
(\mathrm{CO}, \mathrm{TC}, \\
\mathrm{LS})\end{array}$ & $\begin{array}{l}\text { C*40 } \\
(1983 r) \\
\text { Sturidoila- } \\
\text { *epe }\end{array}$ & & $\begin{array}{l}\text { Bergstroemognathus extensus Serpagli [R] } \\
\text { Fahraeusodus marathonensis (Bradshaw) [R] } \\
\text { Juanognathus jaanussoni Serpagli [R] } \\
\text { Oepikodus evae (Lindström) [A] } \\
\text { Protoprioniodus aranda Cooper [R] } \\
\text { Tripodus laevis Bradshaw? [R] } \\
\text { "Scandodus" robustus Serpagli [R] }\end{array}$ & $\begin{array}{l}\text { late Arenig (Upper } \\
\text { O. evae Zone-T. } \\
\text { laevis Zone) }\end{array}$ & 2 \\
\hline $\begin{array}{l}82 \\
(\mathrm{TC}, \mathrm{CO})\end{array}$ & $\begin{array}{l}C^{*}-67 \\
(1983 r)\end{array}$ & & $\begin{array}{l}\text { Cordylodus proavus Müller [R] } \\
\text { Hirsutodontus hirsutus Miller elements [R] } \\
\text { Semiacontiodus nogamii (Miller) [R] } \\
\text { Teridontus nakamurai (Nogami) [R] }\end{array}$ & $\begin{array}{l}\text { very late Late } \\
\text { Cambrian (Middle } \\
\text { C. proavus Zone- } \\
\text { low C. lindstromi } \\
\text { Zone) }\end{array}$ & 4 \\
\hline $\begin{array}{l}83 \\
(\mathrm{CO})\end{array}$ & $\begin{array}{l}\text { "R"K-2 } \\
\text { (1983r) } \\
\text { Yasachkaya } \\
\text { Kuranikh } \\
\text { Arenig }\end{array}$ & & $\begin{array}{l}\text { Drepanoistodus aff. D. basiovalis (Sergeeva) } \\
{[\mathrm{C}]} \\
\text { unassigned coniform }[\mathrm{R}] \\
\text { indets. }[\mathrm{R}]\end{array}$ & $\begin{array}{l}\text { late Arenig- } \\
\text { Llanvirn }\end{array}$ & 4.5 \\
\hline $\begin{array}{l}84 \\
(\mathrm{CO}, \mathrm{TC})\end{array}$ & $\begin{array}{l}\text { Opagobekas } \\
\text { Strelok Creek } \\
2 \mathrm{e}, \mathrm{O}_{1} \\
(1971 \mathrm{r})\end{array}$ & $\begin{array}{l}\text { Omolon massif } \\
\text { Omolon terrane }\end{array}$ & $\begin{array}{l}\text { Cordylodus intermedius Furnish [R] } \\
\text { Cordylodus sp. [C] } \\
\text { Variabiloconus bassleri (Furnish) [R] }\end{array}$ & $\begin{array}{l}\text { earliest Early } \\
\text { Ordovician } \\
\text { (Co. angulatus } \\
\text { Zone-R. } \\
\text { manitouensis } \\
\text { Zone) }\end{array}$ & 4 \\
\hline $\begin{array}{l}85 \\
(\mathrm{CO})\end{array}$ & \begin{tabular}{|l|}
$5 \mathrm{a}$ \\
$(1971 \mathrm{r})$
\end{tabular} & & $\begin{array}{l}\text { Cordylodus spp. [A] } \\
\text { coniform [C] }\end{array}$ & late Late Cambrian & 4 \\
\hline
\end{tabular}




\begin{tabular}{|c|c|c|c|c|c|}
\hline $\begin{array}{l}86 \\
(\mathrm{CO})\end{array}$ & $\begin{array}{l}6 \mathrm{~b} \\
\text { Urdakh } \\
\text { Formation, } \\
\text { Strelok Creek } \\
\text { northwest part } \\
\text { Fore-Kolyma } \\
\text { Uplift }\end{array}$ & & $\begin{array}{l}\text { Cordylodus sp. indet. [A] } \\
\text { Iapetognathus sprakersi Landing [R] } \\
\text { Teridontus nakamurai (Nogami) [A] }\end{array}$ & $\begin{array}{l}\text { earliest Early } \\
\text { Ordovician } \\
\text { (Co. angulatus } \\
\text { Zone- lower part } \\
\text { R. manitouensis } \\
\text { Zone) }\end{array}$ & 4 \\
\hline $\begin{array}{l}87 \\
(\mathrm{CO})\end{array}$ & $\begin{array}{l}5694-1 \\
(1980 r), O_{2} \\
\text { Kuochatskaya } \\
\text { svita } \\
\text { "ya" } 90\end{array}$ & & $\begin{array}{l}\text { cordylodontid }[\mathrm{R}] \\
\text { indets. }[\mathrm{C}]\end{array}$ & \begin{tabular}{|l} 
early Early \\
Ordovician-latest \\
Late Cambrian
\end{tabular} & $5.5-6.5$ \\
\hline $\begin{array}{l}88 \\
(\mathrm{SA}, \mathrm{CO})\end{array}$ & $\begin{array}{l}95-\mathrm{JT}-45 \\
(11364-\mathrm{CO}) \\
66^{\circ} 30.108^{\prime} \mathrm{N} \\
/ \\
171^{\circ} 19.230^{\prime} \mathrm{E}\end{array}$ & $\begin{array}{l}\text { Unnamed unit: } \\
\text { massive black } \\
\text { limestone } \\
\text { containing crinoid } \\
\text { debris of } \\
\text { Ordovician age. } \\
\text { Arctic Alaska- } \\
\text { Chukotka terrane }\end{array}$ & $\begin{array}{l}\text { Acanthodina? sp. indet. [R] } \\
\text { Acanthocordylodus spp. indet. [C] } \\
\text { Drepanoistodus sp. of early Middle Ordovician } \\
\text { morphotype [C] } \\
\text { indets. [A] }\end{array}$ & $\begin{array}{l}\text { Middle } \\
\text { Ordovician, } \\
\text { probably early } \\
\text { Middle } \\
\text { (conodonts poorly } \\
\text { preserved) }\end{array}$ & $\begin{array}{c}\text { chiefly } 6.5 \\
\text { and } 7 \text {, and } \\
\text { lesser } 5 \text { and } \\
5.5\end{array}$ \\
\hline $\begin{array}{c}\text { SAMPLE NO. } \\
\text { (CONODONT } \\
\text { FAUNAL } \\
\text { AFFINITIES) }\end{array}$ & $\begin{array}{l}\text { SECTION AND } \\
\text { FIELD NO. }\end{array}$ & $\begin{array}{c}\text { STRATIGRAPHIC } \\
\text { UNIT, LITHOLOGY, } \\
\text { AND STRUCTURAL } \\
\text { BLOCK }\end{array}$ & CONODONT FAUNA & AGE & CAI \\
\hline
\end{tabular}

